\title{
Los dualismos opresivos y la educación ambiental
}

\author{
ALICIA H. PULEO \\ Universidad de Valladolid
}

RESUMEN. La educación ambiental es considerada un factor clave para la formación de una ciudadanía consciente de la necesidad de avanzar hacia una sociedad sustentable. Sin pretensiones omniexplicativas, este trabajo busca subrayar, desde la perspectiva de género, la persistencia de ciertos dualismos opresivos vinculados al par Cultura-Naturaleza. A partir de su análisis, propone que ciertos valores y actitudes históricamente devaluados y marginalizados como femeninos sean integrados en la elaboración de una educación ambiental a la altura de los retos del siglo XXI.

\section{INTRODUCCIÓN}

Respondiendo a la iniciativa de las Naciones Unidas de instituir a partir de 2005 una Década de la Educación para el Desarrollo Sostenible ${ }^{1}$, el Tercer Seminario Ibérico de Ciencia, Tecnología y Sociedad que ha tenido lugar en junio de 2004 en Aveiro con participantes de España, Portugal y América Latina aprobó un manifiesto titulado Compromiso por la Sostenibili-
ABSTRACT. Environmental education is considered a key factor for the formation of a citizenry conscious of the need to move toward a sustainable society. Without attempting to offer a comprehensive explanation of all aspects of this issue, this article highlight, from the perspective of gender, the persistence of certain oppressive dualisms related to the paired concepts of Culture and Nature. Based on this analysis, the article proposes that certain values and attitudes that historically have been devalued and marginalized as feminine be integrated into a conceptualization of environmental education that takes up the challenges facing us in the twenty-first century.

$\mathrm{dad}^{2}$. Este documento llama a la ciudadanía, y en particular a los educadores, a participar de la iniciativa de Naciones Unidas frente a una crisis ecológica cada vez más evidente. Se menciona la contaminación que envenena el medio ambiente y acarrea el cambio climático, la miseria creciente de miles de millones de seres humanos en algunas partes del planeta mientras una quinta parte de la humanidad practica un consumismo irresponsable, el excesivo 
crecimiento demográfico mundial, el aumento en todos los países de enfermedades relacionadas con la alimentación y la contaminación, las guerras, las «limpiezas étnicas», la explotación infantil y la destrucción de la diversidad cultural.

Para responder desde la filosofía a esta llamada, tenemos que seguir preguntándonos: ¿Qué entendemos por Naturaleza? ¿Qué valores y actitudes nos permitirían avanzar hacia una sociedad sustentable que utilice la tecnología sin caer en tecnolatría, es decir, en una confianza absolutamente ciega hacia ella? ¿Qué creencias no examinadas impiden u obstaculizan ese avance? ¿El problema reside sólo en la falta de aplicación de los criterios éticos debido a intereses egoístas? ¿O, como se ha planteado desde la filosofía medioambientalista, es necesario revisar nuestra visión del mundo y nuestra autodefinición para introducir cambios en lo que se considera valioso y así poder hacer frente a las fuerzas desatadas del nuevo Golem que nos amenaza? Y también podemos plantearnos el siguiente interrogante, sin el cual lo anterior quedaría en letra muerta: ¿Cómo se puede educar eficazmente en la sustentabilidad? ¿Adiestrando en un cálculo de intereses individuales a corto, medio y largo plazo que invite a ajustarse a las tres R (reducir, reutilizar y reciclar)? ¿Informando simplemente sobre las consecuencias de un mal uso de los «recursos»? $¿ \mathrm{O}$ fomentando la solidaridad intraespecífica en el espacio (con los habitantes de las zonas más desfavorecidas del planeta) $\mathrm{y}$ en el tiempo (con las generaciones futuras)? ¿O, más aún, extendiendo nuestra capacidad empática y nuestras obligaciones éticas más allá de nuestra especie?

La crisis ecológica a la que nos enfrentamos responde a una causalidad múltiple que no pretendo ni siquiera llegar a nombrar exhaustivamente en este trabajo. Sólo me propongo señalar algunos aspectos ético-políticos, a mi juicio importantes y por lo general poco tenidos en cuenta. En pri- mer lugar, pasaré revista a algunos conceptos y operaciones propios de lo que se ha dado en llamar la «lógica de la dominación» y posteriormente examinaré cómo subsisten, implícita e inconscientemente, en los esfuerzos pedagógicos por ofrecer una eficaz formación ambiental.

\section{ALGUNOS DUALISMOS DE NUESTRA HERENCIA COMÚN}

En primer lugar, quiero señalar que Naturaleza no es un concepto meramente descriptivo, sino eminentemente político ${ }^{3}$. Designa, en el discurso filosófico hegemónico, la parte inferior del par Naturaleza/Cultura (o Humano/Naturaleza), aquello que no tiene su finalidad en sí mismo, aquello que carece de sacralidad o dignidad, lo que es medio para un fin que le es externo, lo sujeto al devenir cíclico, lo carente de historia y de progreso, lo que puede ser apropiado por el hombre racional e industrioso, lo absurdo en la perspectiva del pesimismo existencial.

Las mujeres estamos en una posición particular con respecto a la Naturaleza. Hemos sido adscritas (y seguimos siéndolo) de alguna manera a ella como al ámbito de lo inferior y subhumano. Nuestras reivindicaciones han sido y siguen estando orientadas en gran medida a huir de la Naturaleza para que se nos reconozca como miembros de pleno derecho de su opuesto: la Cultura, la Humanidad. Por ello, el feminismo surge como la demanda de igualdad de la mitad de la humanidad a la otra $\operatorname{mitad}^{4}$ que injustamente la condenaba a la inmanencia de la Naturaleza al imposibilitarle el acceso a la autonomía.

Otros grupos subalternos han sufrido $\mathrm{y}$ sufren procesos similares. Las teorías de la descolonización de Franz Fanon o Albert Memmi han indagado en la formación de la autoimagen idealizada del opresor y la imagen naturalizada del oprimido. La animalización se ha cernido siempre como una amenaza y una condena ${ }^{5}$ sobre muje- 
res, bárbaros, extranjeros, clases desfavorecidas, indígenas y minorías sexuales).

Pero, en lo que parece a primera vista una paradoja, el análisis crítico de los discursos legitimadores de estas adscripciones interesadas a la Naturaleza han generado instrumentos muy pertinentes para esclarecer y transformar nuestras relaciones, como humanos, con lo no humano. Su adecuación se debe a razones de orden teórico y práctico. Por un lado, como estudio de la lógica del dominio, facilita el conocimiento de los mecanismos de la devaluación y la mitificación. Por otro lado, como ha sido puesto de relieve en la Conferencia Mundial La Mujer en el año 2000: igualdad entre los géneros, desarrollo y paz en el siglo XXI celebrada en Nueva York en el 2000 (Beijing + 5) las mujeres son particularmente afectadas por el deterioro medioambiental ligado a los procesos de globalización. En defensa de sus culturas ligadas a la conservación del medio natural, grupos de indígenas y de mujeres pobres de zonas rurales del Tercer Mundo han puesto en marcha iniciativas de defensa de la Naturaleza frente a un desarrollo sostenido que ha afectado negativamente sus vidas. Así nacieron, entre otros, el movimiento de mujeres Chipko de la India para detener la deforestación del Himalaya y el Green Belt de Kenya, fundado por la activista ecologista Wangari Maathai, premio Nobel 2004, que ha plantado más de veinte millones de árboles en doce países africanos para combatir la desertificación. Finalmente, cabe señalar que algunas teóricas ecofeministas destacan cierta similitud entre las mujeres y otros grupos no dominantes: un sentido del self interconectado con otras formas de vida y más vinculado a una ética del cuidado que a una ética de los derechos entre individuos separados. Si bien este último aspecto de esta teoría es interesante y contiene, a mi juicio, cierto porcentaje de validez descriptiva, ha llevado, en una interpretación tendente al eliminacionismo, a desestimar lo que ha sido y continuará siendo un instrumento imprescindible para la protección de los más vulnerables: el lenguaje de los derechos ${ }^{6}$.

El colectivo femenino y las minorías étnicas han conseguido, si no la igualdad que solicitaban, al menos el reconocimiento de su plena humanidad. Sin embargo, como veremos, continúan afectados por profundos dualismos que hunden sus raíces en un pasado filosófico que rechaza o menosprecia todo aquello que concibe como mera naturaleza.

Estos dualismos estudiados por la filosofía ecofeminista son relaciones de oposición vinculadas a la organización social de sexo-género. Niegan la dependencia con respecto al elemento subordinado e instituyen jerarquías de valor ${ }^{7}$. Se insertan en un sistema de pensamiento anclado en la realidad histórica material de la dominación y la explotación. Una de sus funciones es la legitimación de estas relaciones de poder pero no deben ser pensadas en términos de simple superestructura ideológica ${ }^{8}$. Ellas mismas son tecnologías de dominación, no un mero reflejo de la verdadera realidad, que sería la económica.

De este sistema de pensamiento compuesto de dualismos interconectados con la oposición Cultura/Naturaleza, destacaré algunos particularmente relevantes para nuestro análisis. En primer lugar, evidentemente, el dualismo jerarquizado hombre/mujer que a su vez remite a los dualismos razón/emoción; mentelcuerpo; civilizadol primitivo; espíritu/materia; universal/particular, self/Otro. De todos ellos, al estar relacionados con el imaginario de los sexos, puede decirse que son dualismos generizados. Se construyen por medio de ciertas operaciones características entre las que destacaré, siguiendo a Val Plumwood ${ }^{9}$, las de hiperseparación. denegación, homogeneización, incorporación e instrumentalización.

La hiperseparación, en tanto mecanismo jerarquizador, enfatiza las diferencias, 
negando o minimizando la existencia de rasgos compartidos y rechazando la continuidad. Se trata de una operación bien conocida por los defensores de los animales que cuando creen haber refutado la teoría del abismo ontológico, subrayando las capacidades cognitivas y afectivas no humanas, comprueban que sus adversarios han subido el listón de lo exigido (si se prueba, contra el cartesianismo, que son capaces de sentir dolor, entonces se exigen capacidades lógicas y linguísticas; si se descubre que fabrican instrumentos y son capaces de razonamientos simples y de aprender un lenguaje, entonces se plantea la razón práctica como condición de cualquier derecho, etc.). La conceptualización de los sexos también se ha realizado con recurso a este expediente. Simone de Beauvoir mostró que el «segundo sexo» ha sido concebido como lo Otro con respecto a lo propiamente humano. Así, en la Política ${ }^{10}$, Aristóteles afirma que la razón es lo propio del hombre libre, y que mujeres, animales y esclavos no acceden a la razón, por lo que carecen de libertad y de finalidad en sí mismos. Son para el hombre libre, que es quien determina su finalidad. Aunque el hombre es definido como animal racional, lo propiamente humano será la diferencia específica. La identidad del hombre libre fue determinada por exclusión de las características compartidas con lo dominado: mente frente al cuerpo, razón que controla la emoción, racionalidad que somete a la animalidad interna y externa, espíritu que da forma a la materia (de ahí la teoría de la reproducción humana del Estagirita).

Se constituye y legitima, de esta manera, un grupo con privilegios sobre otro que queda en una posición subalterna. Como bien denunciara Albert Memmi, las identidades de colonizador y colonizado justifican la dominación naturalizándola, convirtiendo lo sociológico e histórico en biológico y metafísico. La animalización persigue al dominado, que intenta deses- peradamente probar su humanidad aprendiendo la lengua y adoptando las costumbres propias del modelo hegemónico de ser humano.

La bipolarización de los sexos con su extrema diferenciación de roles, identidad sexuada y status ha estado conectada durante largos siglos con la oposición Cultura/Naturaleza. La categoría de naturaleza está ligada históricamente en la tradición filosófica occidental a la de $l o$ femenino (pensemos en los hijos mortales que los hombres tienen con las mujeres frente a los hijos eternos de la comunidad de varones en El Banquete de Platón, por ejemplo).

Las mujeres han sido naturalizadas. La antropóloga Sherry Ortner sostuvo que la posición subalterna de las mujeres en el sistema de sexo-género se habría originado en la proximidad de las funciones y tareas de las mujeres con respecto al mundo natural ${ }^{11}$. El embarazo, el parto, la lactancia y una mayor tendencia a la expresión de los sentimientos, así como su participación en tareas de mediación entre naturaleza y cultura como la preparación de los alimentos y la crianza de los niños, de alguna forma habrían contaminado el status del colectivo femenino debido a la devaluación previa de la naturaleza en todas las culturas. Posteriormente, las pretensiones explicativas universales de este artículo fueron recortadas por estudios que demostraron la variación histórica del concepto de Naturaleza y la existencia de algunas culturas etnológicas en las que los varones se identificaban, en tanto cazadores, con la Naturaleza salvaje concebida como superior, y calificaban de «femenina» la cultura que domesticaba animales y plantas ${ }^{12}$. Sin embargo, en todo caso, la hipótesis de Ortner se adapta a la tradición occidental y a gran parte de la oriental. Historiadoras y epistemólogas feministas como Carolyn Merchant ${ }^{13}$ y Evelyn Fox Keller ${ }^{14}$, al estudiar el surgimiento de la ciencia moderna en su lucha contra la 
alquimia, han desvelado el subtexto de género del paradigma baconiano que se presentaba a sí mismo como un proyecto de dominio sobre una Naturaleza concebida como entidad femenina que debía ser seducida y forzada para que, sometida, librara sus secretos. Las metáforas de la agresión sexual y del interrogatorio inquisitorial aplicado a las brujas que encontramos en Bacon no eran elementos de simple retórica, sino expresión de una forma de concebir el estudio de la Naturaleza totalmente contrario a la actitud de empatía que algunas/os científicas/os afirman utilizar en contextos de investigación. Fox Keller compara este paradigma, que llama de objetividad estática, con la metodología de investigación en Genética de Barbara McClintock: «La objetividad dinámica tiende a una forma de conocimiento que garantice la integridad independiente del mundo que nos rodea, al mismo tiempo que sigue siendo consciente de nuestra conectividad con ese mundo, $\mathrm{y}$ de hecho se apoya en ella. En esto, la objetividad dinámica no es diferente de la empatía, una forma de conocimiento de las otras personas que recurre explícitamente a la comunidad de sentimientos y experiencias con el fin de enriquecer nuestro entendimiento del otro/a» ${ }^{15}$.

Las tareas del cuidado de la vida que se realizan en el ámbito familiar son todavía hoy en los países industrializados realizadas en su inmensa mayoría por las mujeres. Aquí podemos ver otros dualismos, propios de la modernidad, pero conectados a los anteriores y apoyados en éstos: los que oponen trabajo productivo y trabajo reproductivo, ámbito público y ámbito doméstico. Esa particular tarea de reproducción de la vida que consiste en el mantenimiento corporal y afectivo de adultos, ancianos y niños sigue siendo prácticamente responsabilidad exclusiva de las mujeres. La actual economía del trabajo productivo reposa en la externalización de los costes medioambientales de la explotación de la Naturaleza no humana y en las tareas domésticas no remuneradas de las mujeres que permiten la renovación de la fuerza de trabajo. El ámbito doméstico aparece, así, como un espacio de Naturaleza en el centro de la cultura. Emerge en segundo plano, como un ámbito inferior cuya función, apenas visible, consiste en permitir que tengan lugar las tareas humanas superiores, que son las únicas que cuentan. Éste es un buen ejemplo del efecto de la operación de denegación característica de los dualismos que examinamos: desde la perspectiva dominante, los servicios del dominado son declarados inesenciales, inferiores. Se niega toda dependencia con respecto a ellos. ¿Y acaso no vivimos también actualmente en la negación de la dependencia de la humanidad con respecto a la Naturaleza como suelo nutricio?

Pero volvamos a los trabajos del cuidado, aquellos que se ocupan de la Naturaleza del ser humano o Naturaleza interna. No es una casualidad si estas actividades carecen de remuneración y se caracterizan por su bajo status. Incluso cuando se convierten en profesiones (por ejemplo, enfermería) continúan marcadas por su origen de género y su cercanía al cuerpo, a la naturaleza y a lo femenino. Tanto las tareas domésticas occidentales como las economías de subsistencia no integradas en el mercado y propias de otras culturas son infravaloradas. Mary Mellor las ha llamado We Economy ${ }^{16}$, economías que satisfacen necesidades vitales materiales y afectivas de la comunidad; muy diferentes en su dinámica a la economía individualista capitalista (My Economy). Esta última, en su búsqueda de expansión indefinida, alienta los procesos de globalización neoliberal responsables de la actual destrucción ecológica a gran escala y de la explotación creciente de grandes grupos humanos.

Sin ignorar los mecanismos puramente económicos actualmente en marcha, el 
análisis de los dualismos de nuestro pensamiento nos puede permitir comprender mejor este fenómeno y generar un pensamiento más propio de una sociedad sustentable. En esta sociedad del futuro, deberá superarse lo que se llama el «perfil masculino del puesto de trabajo» o suposición de que «el trabajador» tiene una mujer que asegura la infraestructura doméstica. Actualmente, el resultado de no haber encarado abiertamente la transformación social exigida por la salida de las mujeres al mundo del trabajo asalariado es la doble y triple jornada que afecta a estas últimas con todos sus efectos negativos (stress, enfermedades, problemas familiares, etc.). Mejorar la calidad de vida implicaría adaptar los tiempos del trabajo asalariado a los tiempos y necesidades vitales de esos organismos vivos que son las personas. La realidad actual está lejos de encaminarse hacia esta solución. El tiempo continúa acelerándose junto con la crisis ecológica ${ }^{17}$.

Observemos que, aún hoy, hablar de mujeres y de trabajo doméstico parece introducir lo particular e inferior en el ámbito superior de lo neutro universal. Ello se debe a que la autoimagen del dominador ha constituido, en palabras de Celia Amorós, un «sujeto inverosímil» o «sujeto iniciático» o «desmadrado», una fantasía de sujeto «inengendrado y generador absoluto de sentido» ${ }^{18}$ que la teoría de las relaciones objetales identifica como una tendencia masculina en las relaciones madre-hijo de las sociedades patriarcales. En el sistema de sexo-género, la afirmación del self se produce en ruptura y contraposición con la madre y con todo lo que ésta representa, fuertemente devaluado: suelo nutricio, cuidados, afectividad, apertura y relación estrecha con los otros. La distancia afectiva y el pensamiento dualista sujetolobjeto forman parte del androcentrismo hegemónico y se hallan ligados al antropocentrismo exagerado de nuestra visión de la Naturaleza.
El par trascendencialinmanencia tematizado por Beauvoir para arrancar a las mujeres de la heterodesignación ilegítima como «Naturaleza» nos ha dejado una herencia emancipatoria que remite a las ideas de libertad, igualdad y justicia imprescindibles en toda empresa de liberación. Pero, también posee fuertes connotaciones androcéntricas y antropocéntricas, que han de ser analizadas a efectos de proceder a la inclusión de lo inicialmente excluido y alcanzar un modelo de ser humano y de sociedad compatibles con la continuidad de la vida en la Tierra.

El lado oscuro del legado remite a la ilusión de autogénesis absoluta y a la negación de todo límite (no sólo de opresión social, sino también de necesidad natural). Esta cara perversa de la trascendencia, potenciada por los intereses económicos, ha conducido a una civilización tecnológica destructiva. La identidad viril conquistadora (forjada históricamente en tanto separación y negación extremas de la madre y de lo femenino) mantiene cierto nexo causal con la ilusión suicida de la civilización de conseguir la independencia absoluta con respecto a la Naturaleza. El yo eligiéndose sin límite alguno implica una separación total con respecto a las bases de la vida. Lo corporal aparece como facticidad, como inmanencia fuertemente devaluada.

Un ejemplo de la ambigüedad del concepto de trascendencia es la definición sartreana del hombre como «ser que no es lo que es». Se trata de un canto a la libertad que ha inspirado gran parte de nuestra vida pero que encierra el desprecio de la Naturaleza externa e interna. Por su énfasis en un rasgo humano parcial, constituye quizás más una consigna apta para procesos de liberación anteriores a la crisis ecológica que una descripción ontológica perfectamente adecuada. Las metáforas fálicas de Sartre señaladas por Michele Le Doeuff ${ }^{19}$ y Toril Moi ${ }^{20}$ no son totalmente independientes del núcleo filosófico de su 
teoría. Podemos considerarlas parte del imaginario androcéntrico del que surge la ilusoria definición de lo humano-masculino como puro espíritu desgajado de la Naturaleza, o Naturaleza autoconsciente destinada a dominar sin límites al Otro o Naturaleza no consciente de sí (por otro lado, descripción inadecuada de lo no humano con los datos etológicos que poseemos actualmente). No obstante, quiero subrayar que una reconciliación con la naturaleza podría y debería conservar la potencia de la caracterización antropológica sartreana como salvaguarda de nuestro margen de libertad con respecto a la opresión social e histórica disfrazada tan a menudo de «natural».

El proceso de homogeneización niega la individualidad a los dominados. Esta operación ha sido perfectamente descrita por la teoría feminista. Me limitaré a recordar el agudo análisis realizado por Celia Amorós al respecto: mientras que los hombres se autodefinen como «iguales» al menos formalmente, las mujeres son pensadas como «idénticas» ${ }^{21}$; la individualidad es una cualidad que depende del poder y quien carece de él es «heterodesignado».

Desde una perspectiva feminista, socialista, antirracista y ecológica, podemos decir que género, raza, clase social y especie, en tanto distribuidores de poder, determinan quién puede ser reconocido como individualidad única, no reemplazable ni reducible a patrones predeterminados.

La incorporación es el proceso por el que el otro es definido como poseedor de cualidades que corresponden a los deseos o necesidades del amo. Una de sus formas es la definición negativa. Se destaca la carencia de ciertas cualidades del dominante que han sido declaradas superiores y se le reconocen defectos o virtudes funcionales. Belleza y sensualidad, o incluso sólo sexualidad, podrían ser claros ejemplos de cualidades adjudicadas tradicionalmente a las mujeres. La racionalidad y el juicio moral, en cambio, eran rasgos considerados superiores; y se negaba que los poseyeran las mujeres ${ }^{22}$, a pesar de los numerosos indicios en sentido contrario.

Un ejemplo extremo de esta operación es la reducción de los animales no humanos a extensión, a carne o a cualquier otra cualidad que interese explotar. Tiene su máximo exponente en la moderna granja factoría. El dolor, la desesperación, la necesidad de moverse o de ser amados no es reconocida como existente. $\mathrm{Ni}$ siquiera son víctimas del odio, sólo del desprecio, de la abstracción y de las cifras de rendimiento por metro cuadrado. Pero estamos ya en la cuarta operación de la lógica del dominio: la instrumentalización.

Como su nombre indica, la instrumentalización es la reducción del dominado a un mero medio para los fines del dominador. La virtud del dominado consiste en la adecuación a dichos fines. Así, cuando las mujeres transgredían las normas de su sexo, eran estigmatizadas como perversas. El negro bueno era el resignado a su condición de esclavo o sirviente solícito. Pensar la Naturaleza únicamente en términos reduccionistas de «recursos» y de «gestión de recursos» equivale a negar su existencia independiente de los humanos. Si bien este enfoque ambientalista puede retrasar el suicidio ecológico de nuestra especie, no es suficiente para un verdadero cambio de rumbo.

Hiperseparación, denegación, incorporación, homogeneización e instrumentalización se conjugan hasta volverse contra los supuestamente privilegiados. Así, la hiperseparación y denegación impiden reconocer nuestro parentesco y dependencia de la Naturaleza. La reducción de las cualidades del oprimido a unas pocas funcionales a la opresión (incorporación) y la instrumentalización (por la que se niegan los fines propios del dominado) terminan instrumentalizando al propio anthropos dominador. Como productores mal informados y desprotegidos, sufrimos enferme- 
dades profesionales debidas a la contaminación; convertidos en consumidores, manipulados por los media y regidos por la ley del mercado, enfermamos alimentándonos con la carne repleta de hormonas y antibióticos de pobres seres atormentados por la ganadería intensiva; y como ciudadanos incapaces de hacer cumplir los Derechos Humanos de tercera generación, asistimos impotentes al cambio climático y a las guerras por el dominio de los recursos. Ya Horkheimer y Adorno ${ }^{23}$ advertían algo similar cuando señalaban los efectos de la dialéctica de la Ilustración en la psicología conductista. Sus laberintos terminaban conduciendo a una concepción mecanicista y manipuladora de los seres humanos.

\section{HACIA UNA EDUCACIÓN AMBIENTAL NO ANDROCÉNTRICA}

A las preguntas iniciales de cómo colaborar desde la filosofía en la década de la Educación para la sociedad sustentable, responderé, entonces, que una de las tareas «epimeteicas» ${ }^{24}$ consiste en revisar nuestra autoconciencia como individuos y como especie, haciendo conscientes los dualismos opresivos que subyacen a nuestro pensamiento, así como las operaciones que los constituyen, reconociendo su génesis histórica como elementos de dominación de género, raza, clase y especie. Superar el sexismo, el racismo, el androcentrismo y el antropocentrismo moral fuerte es tanto una exigencia ética para con los oprimidos como una necesidad vital de la humanidad en tiempos del cambio climático.

Los estudios científicos más recientes, en particular los etológicos y ecológicos, facilitan la visión de nuestra especie como parte de un continuum de la Naturaleza, abriendo dos caminos divergentes: la instrumentalización generalizada a la misma humanidad o la extensión del respeto y la empatía hacia la naturaleza no humana.
Para tomar el segundo rumbo y evitar el primero, algunos sectores de la filosofía ambiental consideran que el conocimiento de otras culturas nos puede ayudar a pensar la relación con la Naturaleza en términos de colaboración y no de dominio y destrucción. Como ya apuntaba Montesquieu con gran ironía en sus Cartas persas a pesar de que escribía en plena cúspide de la autoconfianza occidental, siempre enriquece tomar distancia con respecto a las propias creencias y costumbres y compararlas con las ajenas. Pero, en este siglo XXI, la seducción de lo exótico no nos debe hacer olvidar los elementos emancipatorios que la tradición ilustrada ha ofrecido y continúa ofreciendo a las mujeres ${ }^{25}$. Podría suceder que aceptemos bajo nuevos ropajes prejuicios interesados que hemos combatido y desenmascarado en Occidente.

En la línea de búsqueda de otras fuentes culturales, algunas teóricas ecofeministas ${ }^{26}$ anglosajonas han llamado a un «reencantamiento del mundo» que lo libere de la «jaula de hierro» racionalista. Conviene advertir que no se trata de simples llamadas a un misticismo inoperante, sino a la acción política conjunta de movimientos de mujeres, campesinos, obreros, sindicalistas, indígenas, consumidores, ecologistas y defensores de los animales contra la globalización neoliberal y su proyecto de control total sobre el mundo ${ }^{27}$. En América Latina, Ivonne Gebara promueve el ecumenismo y la defensa de la diversidad biológica y cultural frente al pensamiento único de la globalización neoliberal. Aunque no acepta el término de «reencantamiento», esta teóloga brasileña fustiga el extremo antropocentrismo y androcentrismo de la tradición cristiana que nos hizo «cómplices y legitimadores de la destrucción de bosques, ríos, animales y grupos marginales» ${ }^{28}$ y extiende el amor y el respeto cristianos a todas las criaturas vivas y a los ecosistemas que las sustentan. La trascendencia no se obtiene 
despreciando la materia, por el contrario, es redefinida como inmersión en el misterio de la vida y experiencia de pertenencia a una totalidad. De esta forma, la comunidad de los pobres de la Teología de la Liberación de los años setenta se convierte ahora en comunidad vital ecológica en busca de la ecojusticia, convergiendo en ello con la Etica Global de Hans Küng.

Quizás por mi formación filosófica marcada por el pensamiento ilustrado, el pesimismo decimonónico, el materialismo histórico, el existencialismo, y la curiosidad científica, no puedo dejar de preguntarme: $i$ Es imprescindible el reencantamiento del mundo para poder experimentar y enseñar compasión por los seres sintientes o admiración, solicitud y cuidado ante el espectáculo de la belleza y la complejidad naturales? A mi juicio, la respuesta es negativa.

Conseguir una educación ambiental con verdadera fuerza motivadora es una necesidad imperiosa para evitar encaminarnos a una pesadilla de ciencia ficción, a las ya anunciadas guerras incesantes por el agua y otros recursos escasos en una Naturaleza envenenada. A diferencia de otras situaciones que enfrentó exitosamente la filosofía moral, la gravedad del problema ecológico reside justamente en los reducidos plazos temporales de que disponemos. Como afirma Jorge Riechmann: «causa angustia la escasez de tiempo para reaccionar adecuadamente a las consecuencias de nuestros propios actos: el decurso global del desarrollo tecnocientífico y la marcha de la sociedad industrial se asemejan cada vez más a la carrera de un vehículo fuera de control» ${ }^{29}$. De ahí la importancia de la capacidad pedagógica de una adecuada Educación Ambiental que genere ciudadanos y ciudadanas con reivindicaciones de justicia ecológica y con un modelo de calidad de vida basado en el cuidado de la Naturaleza y en el florecimiento de las capacidades humanas, y no en la acumulación insaciable de objetos industriales.
Ahora bien, la Educación Ambiental predominante en la educación formal y no formal hasta hoy no se halla libre de la nefasta influencia del marco conceptual opresivo examinado anteriormente. $\mathrm{La}$ inmensa mayoría de las propuestas no superaría un examen crítico feminista en este sentido.

Recientemente, algunos textos han comenzado a utilizar lenguaje no sexista y han incorporado alusiones a la situación de las mujeres pobres del Tercer Mundo. Esto es bueno pero no basta. Nos podemos preguntar, por ejemplo, ¿por qué aparece solamente la figura femenina de la víctima cuando se dispone de tantas pioneras en la lucha ecologista individual y colectiva?

Sigue presente el dualismo generizado universal/particular en el que el primer elemento denota lo masculino y lo neutro y el segundo lo femenino y sexuado. En los textos pedagógicos que he consultado, no he encontrado alusiones a los derechos sexuales y reproductivos de las mujeres como reconocimiento de su autonomía, a pesar de que constituyen un importante elemento de la sustentabilidad. Se habla de «presión demográfica» pero no de las trabas que aún encuentran las mujeres en muchos lugares del mundo para decidir sobre sus propios cuerpos. Tampoco hay, por ejemplo, mención alguna a la relación entre ciertos antiguos estereotipos masculinos del dominio y la práctica de la caza deportiva que persigue con armas ultramodernas a los animales silvestres en territorios de supervivencia que el desarrollo económico recorta cada vez más como la balzaciana piel de zapa.

Sólo encontramos información «objetiva» sobre contaminación, gestión de residuos, deforestación, desvinculada por regla general, de emociones éticas y estéticas $^{30}$. Aquí subyace el dualismo generizado razón/emoción. En las guías UNESCO (PNUMA) de formación del profesorado para la enseñanza medioambiental se advierte del peligro de caer en la «sensi- 
blería» y de la necesidad de mantenerse en los límites de la objetividad científica. Ya me he referido a algunas críticas con respecto a dicha objetividad desde la perspectiva de la construcción del género-sexo. Como bien señala Carme Adán ${ }^{31}$, las distintas epistemólogas feministas coinciden en la necesidad de revisar esta noción de objetividad introduciendo la dimensión ético-política. Esto no significa caer en el relativismo. Puesto que toda perspectiva es parcial, la objetividad consistirá en una conjunción de visiones parciales o conocimiento situado.

En los manuales de propedéutica a la Educación Ambiental se suele mencionar la importancia del factor afectivo ${ }^{32}$. Pero, a menudo, este reconocimiento no es más que una declaración de principios sobre la que muchos pasan página inmediatamente. Así, se llega a afirmar, por ejemplo, que la Literatura no es materia apta para la Educación Ambiental ${ }^{33}$. ¿Cómo puede afirmarse esto cuando sabemos que, desde la infancia a la edad adulta, la literatura (y otras narrativas artísticas de mayor o menor valor estético como el cine) nos educan emocional y moralmente? Baste como ejemplo el premio Nobel de Literatura 2003, J. M. Coetzee, que con su escritura magistral y esa mayor libertad que tiene el arte con respecto a la filosofía y las ciencias, con esa capacidad de integrar razón y emoción, superando dualismos opresivos de vieja data, ha llamado en la voz del personaje de la anciana escritora Elisabeth Costello a la «imaginación compasiva» ${ }^{34}$, exponiendo ante nuestra conciencia moral todo el horror de la moderna condición animal.

Ecología Social, perspectiva CTS, Ecología Política y Ecologismo de los Pobres son designaciones de la tendencia de los últimos años a una vinculación de las descripciones propias de las ciencias naturales con la problemática socio-política de la desigualdad económica y la injusticia. Pero esta mejora del enfoque no implica necesariamente una superación de los dualismos opresivos examinados ${ }^{35}$. Algunos pedagogos de Educación Ambiental hacen referencias elogiosas a las investigaciones de Carol Gilligan sobre «la otra voz» en la ética, pero, una vez hecho esto, olvidan rápidamente la importancia del pathos vinculado a los valores del cuidado ${ }^{36}$.

Dejando la «formación de formadores» en Educación Ambiental y volviendo a los textos y desarrollos curriculares dirigidos a la niñez y la adolescencia, podemos comprobar que, por lo general, persisten las operaciones de homogeneización, hiperseparación e incorporación que reducen a todos los seres vivos no humanos (hasta los emocionalmente más cercanos a quienes se quiere educar) a «medio ambiente», «especies», «recursos», «fauna» o «Naturaleza».

$\mathrm{El}$ «hombre» parece habitar un extraño mundo vacío en el que no hay más individuos que los humanos en «ecosistemas» que pueden alterarse si no se lleva a cabo una «gestión racional de los recursos». Este profundo prejuicio androcéntrico y antropocéntrico fuerte que desprecia como «sensiblería» los sentimientos empáticos hacia los animales, las plantas y el resto de la Naturaleza me parece poco apto para suscitar un cambio de valores y de actitudes en las nuevas generaciones ${ }^{37}$. En el discurso hegemónico de la Educación Ambiental existe un subtexto de género que exige la separación y el dominio con respecto a lo considerado "Naturaleza». Consciente o inconscientemente, la emoción, la benevolencia, la empatía son considerados poco científicos, femeninos, y son reprimidos como Naturaleza inferior. Como mucho, se suele preconizar el «respeto», virtud severa que mantiene las distancias («respeto por los animales del entorno» ${ }^{38}$, «respeto a toda forma de vida»). Dado que no se trata del exigente respeto kantiano a las personas, nos podemos preguntar a qué queda reducido ese respeto cuando no se acompaña de la imaginación compasiva de que nos hablaba 
Coetzee y se recomienda animar al alumnado, en cambio, a participar y recuperar fiestas, tradiciones y costumbres ${ }^{39}$ sin establecer diferencias entre aquellas que ayudan a una nueva conciencia ecológica y las que, por el contrario, refuerzan las convicciones dualistas opresivas.

¿Es posible desplazar la pasión del dominio con detallados cálculos sobre la gestión de los recursos y los residuos? ¿Despertaremos el interés de niñas, niños y jóvenes rechazando por «sensiblera» su frecuente tendencia a empatizar con otros seres vivos? Sólo la unión de conocimientos y sentimientos de benevolencia puede ser una pedagogía eficaz en la educación ambiental. No basta la razón instrumental constituida a partir de la exclusión de la afectividad que nos une al Otro. Han de unirse los conocimientos científicos, la solidaridad y la compasión ${ }^{40}$.

Para tal fin, la utilidad del análisis ecofeminista consiste en aportar claves de género sobre nuestra relación con la naturaleza externa e interna, con el Otro no humano y con las pulsiones humanas y su modelado. Como ha destacado María Xosé Agra, «los ecofeminismos tienen la tarea y el reto, no de salvar el mundo, sino de ayudarnos a pensar y actuar de otra manera con relación a la naturaleza y a las mujeres, lo cual no significa rechazar la filosofía, la ciencia, la racionalidad» ${ }^{41}$.

La Educación Ambiental no puede superar los dualismos generizados y jerarquizados con la simple utilización de terminaciones gramaticales masculinas y fe- meninas o la mención de las mujeres como primeras víctimas de la crisis ecológica en el Tercer Mundo. Éste es un buen comienzo, pero queda mucho por andar. Tendrá que cuestionar estereotipos de género que ya no son adaptativos a esta altura de la Historia y de la evolución de nuestra especie. Habrá de interesarse por los planteamientos de la educación para la igualdad entre los sexos y de la educación para la paz, para que la interdependencia de estos enfoques no quede en una simple mención inicial sin un verdadero desarrollo integrado. Y deberá saberse conjugar armoniosamente razón y pasión, justicia y cuidado, metas pedagógicas cognitivas y afectivas.

Propongo, pues, junto a la información científica transdisciplinaria imprescindible para una correcta comprensión y eventual solución de los problemas ecológicos de la sociedad industrial y globalizada, una $e d u$ cación sentimental y moral a partir del rechazo de los dualismos opresivos generizados y en base a la aceptación como plenamente humanos de actitudes empáticas y valores del cuidado, hasta hoy todavía devaluados y marginalizados por su relación con la identidad femenina, tal como, al menos, ésta se ha definido históricamente.

Una Educación Ambiental que haya realizado este paso será, no sólo más eficaz en la formación de una joven ciudadanía ecológicamente responsable, sino que habrá avanzado hacia un concepto de lo humano que integre el sentir y las experiencias históricas de las mujeres y de los hombres en un pie de igualdad.
1 Sobre la educación ambiental basada en la definición de desarrollo sostenible de la Conferencia de Río y en los Derechos Humanos de tercera generación, véase Francisco Aramburu, «Ética y Educación Ambiental», en José María García Gómez-Heras y Carmen Velayos, Tomarse en serio la Naturaleza. Ética ambiental en perspectiva multidisciplinar, Madrid,
Biblioteca Nueva, 2004. Para una crítica del concepto de «desarrollo sostenible», véase Joan Martínez Alier, El ecologismo de los pobres. Conflictos ambientales y lenguajes de valoración, Barcelona, Icaria, 2004.

${ }^{2}$ Revista Eureka sobre Enseñanza y Divulgación de las Ciencias, núm. 3, vol. I, 2004. Este Compromiso puede también consultarse en la Red. 
${ }^{3}$ Sobre el carácter político del concepto de Naturaleza, véase Barbara Holland-Cunz, Ecofeminismos, trad. Arturo Parada, Madrid, Ed. Cátedra, 1996.

${ }^{4}$ Así lo entendían, ya en 1825, William Thompson y Anna Wheeler cuando titularon su obra La demanda de la mitad de la raza humana, las mujeres, contra la pretensión de la otra mitad, los hombres, de mantenerlas en la esclavitud política y, en consecuencia civil y doméstica (introducción de Ana de Miguel Comares, 2000)

5 Véase Armelle Le Bras Chopard, Le zoo des philosophes. De la bestialisation à l' exclusion, París, Plon, 2000. Hay traducción castellana en Taurus, 2002. E el curso del año 2005, en el debate suscitado por la aprobación de la Ley española del matrimonio homosexual en el Congreso, para combatir esta ampliación de los derechos a este grupo, hubo quien comparó públicamente la relación homosexual con la zoofilia.

6 Sobre esta cuestión, Alicia H. Puleo, «Derechos versus contextualismo: Personas, simios y la ética ecofeminista», Revista de Filosofia de la Universidad de La Laguna, núm. 7, 2000, pp. 353-357; Anna Charlton (1999), "Las mujeres y los animales», en Teorema. Revista Internacional de Filosofía, vol. XVII/3, 1999, pp. 103-116. Para una discusión sobre el status moral de los animales en la ética, véa se Marta Tafalla (ed.), Los derechos de los animales, Barcelona, Idea Books, 2004; Carmen Velayos, «Los derechos de los animales: un reto para la ética", en Jorge Riechmann (coord.), Ética Ecológica. Propues tas para una reorientación, Montevideo, Unuguay, ed. Nordan-Comunidad, 2004, pp. 135-143; Jorge Riechmann, Todos los animales somos hermanos. En sayos sobre el lugar de los animales en las sociedades industrializadas, Madrid, Los libros de la Catarata, 2005.

${ }^{7}$ Karen Warren, «The Power and the Promise of Ecological Feminism», en K. Warren, Ecological Fe minist Philosophies, Indiana University Press, 1996 pp. 19-41.

8 Val Plumwood, Feminism and the Mastery of Nature, Londres-Nueva York, Routledge, 1993.

${ }^{9}$ Ibid.

10 Libro I, caps. II, IV y V

1 Sherry Ortner, «乙Es la mujer con respecto al hombre lo que la naturaleza con respecto a la cultura?», en O. Harris y K. Young, Antropología y feminismo, Barcelona, Anagrama, 1979, pp. 109-131.

${ }_{12}$ Marilyn Strathern (ed.), Nature, Culture and Gender, Cambridge University Press, 1980.

${ }^{13}$ Carolyn Merchant, The Death of Nature, Londres, Wildwood House, 1980.

14 Evelyn Fox Keller, Reflexiones sobre género y ciencia, Ana Sánchez (trad.), Valencia, Ed. Alfons el Magnanim, 1991.

is Ibid., pp.126-127.

16 Mary Mellor, Feminism and Ecology, Nueva York, Polity Press, Cambridge University Press, 1997.
17 Jorge Riechmann, Tiempo para la vida. La crisis ecológica en su dimensión temporal, Málaga, ed. del Genal, 2003.

${ }_{18}$ Celia Amorós, Tiempo de feminismo. Sobre feminismo, proyecto ilustrado y postmodernidad, Madrid, Cátedra, 1997, pp. 21-22.

19 'Michèle Le Doeuff, L'étude et le rouet. Des femmes, de la philosophie, París, Seuil, 1989.

20 Toril Moi, Simone de Beauvoir. Conflits d'une intellectuelle, Préface de Pierre Bourdieu, París, Diderot éditeur, 1995

${ }^{21}$ Celia Amorós, op.cit., p. 211.

${ }_{22}$ Para el paradigmático caso de Kant, véase «El reino de los fines y su gineceo: las limitaciones del universalismo kantiano a la luz de sus concepciones antropológicas», en Roberto Aramayo, Javier Muguerza y Antonio Valdecantos (compiladores), El individuo y la historia. Antinomias de la herencia moderna, Barcelona, Paidós, 1995.

23 Max Horkheimer y Theodor Adorno, Dialéctica de la Ilustración. Fragmentos filosóficos, Madrid, Trotta, 2001, p. 291.

${ }_{24}$ Así califica Javier Muguerza las tareas animadas, no ya por la confianza filosófica prometeica en la erradicación total y final del mal y la injusticia, sino en «la esperanza más humilde de que siempre nos será dado luchar en pro de lo que creamos bueno y justo 0 , cuando menos, en contra de lo que creamos malo e injusto», J. Muguerza, « $z$ Convicciones $y / o$ responsabilidades? (Tres perspectivas de la ética en el siglo XXI)», en Revista Laguna, núm. 11, septiembre 2002, pp. 42-43.

25 Véase Celia Amorós, «Presentación (que intenta ser un esbozo del status quaestionis)», en C. Amorós, (ed.), Feminismo y Filosofia, Madrid, Síntesis, 2000, pp. 9-112.

26. Algunas pensadoras ecofeministas se inclinan por reelaboraciones del biocentrismo originario de los indígenas norteamericanos. Tal es el caso de las ya citadas Val Plumwood y Karen Warren. Vandana Shiva ve en la cosmovisión tradicional de su país, la India, una visión más armónica de los humanos en la Naturaleza (Abrazar la vida. Mujer, ecología y desarrollo, Instituto del Tercer Mundo de Montevideo, Uruguay (trad.), Cuademos inacabados 18 , Madrid, ed. horas y HORAS, 1995, y Cosecha robada. El secuestro del suministro mundial de alimentos, Barcelona, Paidós, 2003).

${ }^{27}$ A este tipo de alianzas puntuales llama Val Plumwood en «Artemisa versus Gaiạ», en María Luisa Cavana, Alicia Puleo y Cristina Segura (coords.), Mujeres y Ecología: Historia, Pensamiento, Sociedad, Madrid, Almudayna, 2004, pp. 53-106.

28 I. Gebara, Intuiciones ecofeministas. Ensayo para repensar el conocimiento y la religión, Graciela Pujol (trad.), Madrid, Trotta, 2000, p. 121.

29 Jorge Riechmann, Un mundo vulnerable. Ensayos sobre ecología, ética y tecnociencia, Madrid, Los Libros de la Catarata, 2000, p. 167. 
30 Sobre la necesidad de una «ética estética» para hacer frente al desafío medioambiental, se ha pronunciado en reiteradas ocasiones Ana P. Noguera de Echeverri, coordinadora del Grupo de Trabajo Académico en Pensamiento Ambiental de Colombia. Ver, Noguera, A. P., El reencarnamiento del mundo, Programa de las Naciones Unidas para el Medio Ambiente. PNUMA-Oficina Regional para América Latina y el Caribe, Universidad Nacional de Colombia, 2004.

31 Carme Adán, Feminismo e coñecemento. Da experiencia das mulleres ao cíborg, A Coruña, Espiral Maior Universitas, 2003, p. 253.

32 María Novo, La educación ambiental. Bases éticas, conceptuales y metodológicas, Madrid, Universitas, 1996.

33 Harold Hungerford, Trudi Volk, Dixon Billy, Thomas Marcinkowski, Archibald Sia y Shahrir Jamaluddin, Programa de introducción de la educación ambiental en la formación de profesores de enseñanza primaria, UNESCO-PNUMA, Libros de la Catarata, 1996.

${ }^{34}$ J. M. Coetzee, Elisabeth Costello, Barcelona, Mondadori, 2004, p. 87.

35 Así, Joan Martínez Alier (http://www.ecotropia.com/d1010903) insiste, con razón, en la necesidad de que la educación ambiental sea transdisciplinaria, pero la reduce a una combinación de ciencias naturales y sociales, desterrando los valores éticos y las emociones, en un comprensible deseo de objetividad inexpugnable frente al escepticismo moral reinante en las sociedades de consumo. La motivación para el aprendizaje provendrá, sostiene, de que a través de la transdisciplinaridad descubrirán «que todo encaja», en una palabra, el autor parece aquí confiar sólo en el placer intelectual de la explicación científica aunque su propia posición tiene un claro fundamento ético, perceptible en su obra.
${ }^{36}$ Así lo hace Michael Caduto del Instituto de Ciencias Naturales de Woodstock, Vermont, USA, en su Guía para la enseñanza de valores ambientales, Los Libros de la Catarata, 1992. Previene contra prejuicios étnicos o raciales en el material pero olvida el prejuicio sexista.

37 Un buen ejemplo de la posibilidad de combinar objetividad científica y formación de los sentimientos morales hacia los demás seres vivos en la niñez es el libro de David Suzuki y Barbara Hehner Exploremos el entorno [Barcelona, P. Mayoral y C. Chinchilla (trads.), Labor, 1990]. Las excelentes explicaciones de la cadena trófica y de los procesos bioquímicos naturales se acompañan de una delicada sensibilidad hacia todos los seres vivos. Así, por ejemplo, se recomienda devolver a los insectos al mismo sitio en que estaban antes de la observación; los árboles son presentados como «gigantes» que albergan innumerables seres vivos...

38 Manuel Rico Vercher, El aprendizaje de valores en educación ambiental, Madrid, Ministerio de Obras Públicas y Transportes, Secretaría de Estado para las Políticas del Agua y el Medio Ambiente, 1992.

${ }^{39}$ María José Jiménez Armesto y Laura Laliena Andreu, Educación Ambiental, Madrid, Ministerio de Educación y Ciencia, Secretaría de Estado de Educación, 1992.

40. Ésta es la clave del programa educativo Roots \& Shoots puesto en marcha por Jane Goodall para formar desde alumnado de primaria hasta el de Universidad. Para una explicación bien argumentada de sus fundamentos, véase Jane Goodal y Marc Bekoff, Los diez mandamientos para compartir el planeta con los animales que amamos, Barcelona, Paidós, 2003.

41 María Xosé Agra, «Introducción», en M. X. Agra (comp.), Ecología y feminismo, Granada, Comares, 1997, p. 20. 